\title{
Shallow Water Wave Models with and without Singular Kernel: Existence, Uniqueness, and Similarities
}

\author{
Emile Franc Doungmo Goufo ${ }^{1}$ and Sunil Kumar ${ }^{2}$ \\ ${ }^{1}$ Department of Mathematical Sciences, University of South Africa, Florida 0003, South Africa \\ ${ }^{2}$ Department of Mathematics, National Institute of Technology, Jamshedpur 831014, India \\ Correspondence should be addressed to Emile Franc Doungmo Goufo; dgoufef@unisa.ac.za
}

Received 19 September 2016; Accepted 27 November 2016; Published 6 February 2017

Academic Editor: Qin Yuming

Copyright (c) 2017 Emile Franc Doungmo Goufo and Sunil Kumar. This is an open access article distributed under the Creative Commons Attribution License, which permits unrestricted use, distribution, and reproduction in any medium, provided the original work is properly cited.

\begin{abstract}
After the recent introduction of the Caputo-Fabrizio derivative by authors of the same names, the question was raised about an eventual comparison with the old version, namely, the Caputo derivative. Unlike Caputo derivative, the newly introduced CaputoFabrizio derivative has no singular kernel and the concern was about the real impact of this nonsingularity on real life nonlinear phenomena like those found in shallow water waves. In this paper, a nonlinear Sawada-Kotera equation, suitable in describing the behavior of shallow water waves, is comprehensively analyzed with both types of derivative. In the investigations, various fixed-point theories are exploited together with the concept of Piccard $\mathscr{K}$-stability. We are then able to obtain the existence and uniqueness results for the models with both versions of derivatives. We conclude the analysis by performing some numerical approximations with both derivatives and graphical simulations being presented for some values of the derivative order $\gamma$. Similar behaviors are pointed out and they concur with the expected multisoliton solutions well known for the Sawada-Kotera equation. This great observation means either of both derivatives is suitable to describe the motion of shallow water waves.
\end{abstract}

\section{Introduction}

The concept of derivative with generalized order has been intensively used to model many real life phenomena with the general formulation given in many works [1-4]. The version of Caputo fractional derivative (CD) [5] remains the most popular thanks to its useful properties suitable to describe real life phenomena [5-10], especially in those phenomena related to the dynamics of diseases [11-14]. However, to address the issues caused by the singular kernel noticed in Caputo fractional derivative, Caputo and Fabrizio recently proposed another fractional derivative with no singularity called the Caputo-Fabrizio fractional derivative (CFD). Since then, many authors used the CFD to solve some real world problems as shown in some works $[7,15,16]$. The CFD was modified in [7] to generate the related RiemannLiouville version. Recall that the Caputo-Fabrizio fractional derivative reads as the convolution of the local derivative and the function exponential. The concept was generalized by introducing the two-parameter fractional derivative with nonlocal and nonsingular kernel $[17,18]$, like, for instance, the Goufo-Caputo derivative with two parameters and the Goufo-Riemann-Liouville derivative with two parameters proven to have a huge impact in Chaos theory and image processing. Investigations on fractional derivatives like the CFD are still ongoing around the world and this paper follows the same momentum by providing an analysis of the SawadaKotera equation within the scope of both Caputo and CaputoFabrizio derivatives.

It is important to notice that the Sawada-Kotera equation is a nonlinear differential equation that falls into the class of Kortewegde-de Vries- (KdV-) type equations. It has applications in waves theory, like, for instance, in shallow water waves, ion-acoustic waves, or stratified internal waves. It is also classified in the hierarchy of equations suitable to be solved via Lax operator [19-21]. Hence, the nonlinear 
Sawada-Kotera type model is comprehensively investigated in this work using the CD and CFD defined, respectively, by

$$
{ }^{c} D_{t}^{\gamma} f(x, t)=\frac{1}{\Gamma(1-\gamma)} \int_{0}^{t}(t-\xi)^{-\gamma} \frac{d}{d \xi} f(x, \xi) d \xi,
$$

$0<\gamma \leq 1$, and

$$
\begin{aligned}
{ }^{c f} D_{t}^{\gamma} f(x, t) & \\
\quad & =\frac{(2-\gamma) M(\gamma)}{2(1-\gamma)} \int_{0}^{t} \frac{d}{d \xi} f(x, \xi) \exp \left(-\frac{\gamma(t-\xi)}{1-\gamma}\right) d \xi
\end{aligned}
$$

$0<\gamma \leq 1$, where $M(\gamma)$ is a normalization function such that $M(0)=M(1)=1$.

The well-known antiderivative associated with the $\mathrm{CD}$ is given as

$$
I_{0}^{-\gamma} f(x, t)=\frac{1}{\Gamma(\gamma)} \int_{0}^{t} \frac{f(x, \xi)}{(t-\xi)^{1-\gamma}} d \xi
$$

while the antiderivative associated with the CFD is defined as $[15,16]$

$$
\begin{aligned}
{ }^{c f} I_{t}^{\gamma} f(x, t)= & \frac{2(1-\gamma)}{(2-\gamma) M(\gamma)} f(x, t) \\
& +\frac{2 \gamma}{(2-\gamma) M(\gamma)} \int_{0}^{t} f(x, \xi) d \xi,
\end{aligned}
$$

$\gamma \in[0,1], t \geq 0$. The latter antiderivative when applied to a function $f$ gives a sort of average between the function and its first-order integral.

As we mentioned earlier, the CFD is different form the $\mathrm{CD}$ in the sense that it presents a kernel with no singularity at $t=\xi$. Furthermore, the following relations hold for the CFD:

$$
\begin{aligned}
& \lim _{\gamma \rightarrow 1}{ }^{c f} D_{t}^{\gamma} f(x, t)=\frac{\partial}{\partial t} f(x, t), \\
& \lim _{\gamma \rightarrow 0}{ }^{c f} D_{t}^{\gamma} f(x, t)=f(x, t)-f(x, 0) .
\end{aligned}
$$

\section{Analysis Using the Caputo Fractional Derivative (CD)}

Existence of the exact solution of the nonlinear SawadaKotera equation modeled with Caputo time fractional derivative is proven in this section. Recall that similar analysis was performed in other works $[22,23]$. We focus our attention on the following Sawada-Kotera model expressed as

$$
\begin{aligned}
{ }^{C} D_{t}^{\gamma} f(x, t)= & -f_{x x x x x}(x, t)-15 f(x, t) f_{x x x}(x, t) \\
& -15 f_{x}(x, t) f_{x x}(x, t) \\
& -45 f^{2}(x, t) f_{x}(x, t),
\end{aligned}
$$

subject to the initial condition

$$
f(x, 0)=\zeta(x),
$$

where ${ }^{C} D_{t}^{\gamma} f(x, t)$ represents the CD as defined in (1). The first step to follow in this analysis is to transform (6) into an integral equation. Hence, we apply on both sides the antiderivative (3) to get

$$
\begin{aligned}
& f(x, t)-f(x, 0)=\frac{1}{\Gamma(\gamma)} \int_{0}^{t}(t-y)^{\gamma-1}\left(-f_{x x x x x}(x, t)\right. \\
& -15 f(x, t) f_{x x x}(x, t)-15 f_{x}(x, t) f_{x x}(x, t) \\
& \left.\quad-45 f^{2}(x, t) f_{x}(x, t) d y\right) .
\end{aligned}
$$

For reasons of simplicity, the following operator is considered:

$$
\begin{aligned}
J(x, t, f)= & -f_{x x x x x}(x, t)-15 f(x, t) f_{x x x}(x, t) \\
& -15 f_{x}(x, t) f_{x x}(x, t) \\
& -45 f^{2}(x, t) f_{x}(x, t) .
\end{aligned}
$$

The next stage is to prove that the Lipschitz condition with respect to $f$ holds for the operator $J(x, t, f)$. Thus,

$$
\|J(x, t, f)-J(x, t, g)\|=\| \begin{gathered}
(g-f)_{x x x x x}(x, t)+15 g(x, t) g_{x x x}(x, t)-15 f(x, t) f_{x x x}(x, t) \| \\
15 g_{x x}(x, t) g_{x}(x, t)-15 f_{x x}(x, t) f_{x}(x, t) \\
45 g^{2}(x, t) g_{x}(x, t)-45 f^{2}(x, t) f_{x}(x, t)
\end{gathered}
$$

Exploiting the norm's properties, the above yields

$$
\begin{aligned}
& \|J(x, t, f)-J(x, t, g)\| \leq\left\|(g-f)_{x x x x x}(x, t)\right\| \\
& \quad+\left\|15 g(x, t) g_{x x x}(x, t)-15 f(x, t) f_{x x x}(x, t)\right\| \\
& \quad+\|-15(f)_{x x}(x, t) f_{x}(x, t) \\
& \quad+15(g)_{x x}(x, t) g_{x}(x, t)\|+\|-45 f^{2}(x, t) f_{x}(x, t) \\
& \quad+45 g^{2}(x, t) g_{x}(x, t) \| .
\end{aligned}
$$

Exploiting the Lipschitz condition for the standard derivative function, there are positive constants $A, B, C, \psi_{1}, \psi_{2}, \psi_{3}, \psi_{4}$, $\psi_{5} ; \vartheta_{1}, \vartheta_{2}, \vartheta_{3}$; and $\mu_{1}$ and $\nu_{1}$ such that

$$
\begin{aligned}
& \left\|(g-f)_{x x x x x}(x, t)\right\| \leq \psi_{1} \psi_{2} \psi_{3} \psi_{4} \psi_{5}\|f-g\|, \\
& \left\|15 g(x, t) g_{x x x}(x, t)-15 f(x, t) f_{x x x}(x, t)\right\| \\
& \quad \leq 15 \vartheta_{1} \vartheta_{2} \vartheta_{3} A\|f-g\|,
\end{aligned}
$$




$$
\begin{aligned}
& \left\|25(f)_{x x}(x, t) f_{x}(x, t)-25(g)_{x x}(x, t) g_{x}(x, t)\right\| \\
& \quad \leq \frac{25}{2} \mu_{1} \mu_{2} B\|f-g\|, \\
& \left\|-45 f^{2}(x, t) f_{x}(x, t)+45 g^{2}(x, t) g_{x}(x, t)\right\| \\
& \quad \leq \frac{45}{3} v_{1} v_{2} C\|f-g\| .
\end{aligned}
$$

Substituting (12) into (11) leads to

$$
\begin{aligned}
& \|J(x, t, f)-J(x, t, g)\| \leq\left(\psi_{1} \psi_{2} \psi_{3} \psi_{4} \psi_{5}\right. \\
& \left.\quad+15 \vartheta_{1} \vartheta_{2} \vartheta_{3} A+\frac{25}{2} \mu_{1} \mu_{2} B+15 v_{1} v_{2} C\right)\|f-g\| .
\end{aligned}
$$

Taking

$$
\begin{aligned}
\mathscr{K}= & \psi_{1} \psi_{2} \psi_{3} \psi_{4} \psi_{5}+15 \vartheta_{1} \vartheta_{2} \vartheta_{3} A+\frac{25}{2} \mu_{1} \mu_{2} B \\
& +15 v_{1} \nu_{2} C .
\end{aligned}
$$

thus

$$
\|J(x, t, f)-J(x, t, g)\| \leq \mathscr{K}\|f-g\|,
$$

Hence, the desired Lipschitz conditions are proven. Making use of the results above, it is possible to evaluate the quantity

$$
\|f(x, t)\|, \quad t \in[0, T] .
$$

Considering the fact that we are analyzing a real world phenomenon, we must assume existence of initial conditions that are bounded, whence

$$
\begin{aligned}
\|f(x, t)\| \leq & \|f(x, 0)\| \\
& +\frac{1}{\Gamma(\gamma)} \int_{0}^{t}(t-y)^{\gamma-1} J(x, y, f(x, y)) d y \\
\leq & \|f(x, 0)\|+\frac{\mathscr{K}}{\Gamma(\gamma)} \int_{0}^{t}(t-y)^{\gamma-1} d y \\
= & B+\frac{\mathscr{K} T^{\gamma}}{\gamma \Gamma(\gamma)}
\end{aligned}
$$

which gives following theorem.

Theorem 1. Take $0<\gamma<1$ and assume that $\zeta$ given in (7) is a bounded function. Let $J[x, t, f(x, t)]:[[a, b] \times[0, T]] \times E \rightarrow R$ (with $R \supset E$ ) be a continuous with respect to $t$ for any $x$ fixed in $[a, b], f \in \bar{E}$.

If $f(x, t) \in C^{0}[[a, b] \times[0, T]]$, then $f(x, t)$ satisfies model (6) if and only if $f(x, t)$ satisfies the corresponding Volterra integral model (8).

Proof. We start with the proof of the necessity condition by assuming that $f(x, t) \in C^{0}[[a, b] \times[0, T]]$ verifies model (6). Since $J(x, y, f(x, y)) \in C[[a, b] \times[0, T] \times Y]$ for all $f \in \bar{Y}$, then (6) means that it is possible to find the Caputo fractional derivative of $f$ in $C[[a, b] \times[0, T]]$. However

$$
{ }_{0}^{C} D_{t}^{\gamma} f=\frac{\partial}{\partial t}\left(I_{0}^{-\gamma}\right)[f(x, t)-f(x, 0)] .
$$

Exploiting existing results in [9] for $\vartheta=0$, then

$$
I_{0}^{-\gamma}[f(x, t)-f(x, 0)] \in C^{0}[[a, b] \times[0, T]] .
$$

Again applying the results in [9] for $\vartheta=0$ to

$$
V(x, t)=f(x, t)-f(x, o),
$$

then we obtain

$$
\begin{aligned}
I_{0}^{\gamma}{ }_{0}^{C} D_{t}^{\gamma} f(x, t) & =I_{0}^{\gamma}{ }_{0}^{C} D_{0}^{\gamma}[f(x, t)-f(x, 0)] \\
& =f(x, t)-f(x, 0)-\sum_{j=1}^{1} \frac{f_{1-\gamma}^{i-j}(x, 0)_{\gamma-j}}{\Gamma(\gamma-j+1)}
\end{aligned}
$$

with $f_{1-\gamma}(x, t)=I_{0}^{1-\gamma}[f(x, t)-f(x, 0)]$. Integration by parts performed in (21), followed by differentiating the resulting expression, leads to

$$
f_{1-\gamma}^{(1-j)}(x, t)=\frac{\partial}{\partial t}\left(I_{0}^{2-\gamma}\left[\partial_{t} f(x, t)-f(x, 0)\right]\right) .
$$

Changing of variable with $t=\delta+\sigma(y-\delta)$ yields

$$
\begin{aligned}
& f_{1-\gamma}^{(1-j)}(x, t) \\
& \quad=\frac{(y-\delta)^{1-\gamma}}{\Gamma(1-\gamma)} \int_{0}^{1}(1-\sigma)^{-\gamma}\left(f^{1-j}[\delta+\sigma(y-\delta)]\right) .
\end{aligned}
$$

Obviously, $0<\gamma<1$ and $f^{1-j}(x, t) \in C[[a, b] \times[0, T]]$. Then, models (23) and (21) take the form

$$
I_{0}^{\gamma}{ }_{0}^{C} D_{t}^{\gamma} f(x, t)=f(x, t)-f(x, 0) .
$$

Because of $I_{0}^{\gamma} J(x, \xi, f(x, \xi)) \in C^{0}[[a, b] \times[0, T]]$ and exploiting the Lipschitz condition of $J$, we get

$$
\left\|I_{0}^{\gamma} J(x, \xi, f(x, \xi))\right\|_{C^{0}[[a, b] \times[0, T]]} \leq \frac{\mathscr{K} T^{\gamma}}{\gamma \Gamma(\gamma)} .
$$

However, applying $I_{0}^{\gamma}$ on both sides of (6) and considering the initial condition, we obtain the desired Volterra version, which proves the necessity condition.

Conversely, we prove the sufficient condition by assuming that $f(x, t) \in C[[a, b] \times[0, T]]$ verifies the Volterra version of (6). We have to show that the initial condition of (6) holds for $f(x, t)$. If we differentiate both sides of the Volterra version, then

$$
\partial_{t} f(x, t)=\frac{1}{\Gamma(\gamma-1)} \int_{0}^{t} J(x, \xi, f(x, \xi))(t-\xi)^{\gamma} d \xi .
$$


The similar change of variable $t=\delta+\sigma(y-\delta)$ applied in the Volterra version for $k=1$ yields

$$
\begin{aligned}
& f^{k}(x, t)=\frac{(y-\delta)}{\Gamma(\gamma-k)} \\
& \quad \cdot \int_{0}^{t} \frac{J(x, \delta+\sigma(y-\delta), f(x, \delta+\sigma(y-\delta)))}{(1-\delta)^{1-\gamma+k}} d y .
\end{aligned}
$$

Considering the limit as $y \rightarrow \delta^{+}$and exploiting the fact that $J$ is continuous prove that the required the initial conditions hold, which shows the sufficiency condition.

Theorem 2. Since the condition $0<\gamma<1$ is satisfied and the Lipschitz condition holds for the operator J, there is a unique solution for model (8) in the space

$$
C^{0, \gamma}[0, T] \times[a, b] .
$$

Proof. The proof begins by proving that it is possible to find a unique solution $f(x, t)$ of model $(6)$ in $C[[a, b] \times[0, T]]$. By Theorem 2 it will be sufficient to point out the existence of a unique solution $f(x, t) \in C^{0}[[a, b] \times[0, T]]$ of the nonlinear Volterra integral equation (8). Recall that (8) makes sense when it is applied in any interval $[0, \xi] \subseteq[0, T]$. Let us then choose an appropriate $t_{1} \in[0, T]$ such that

$$
\left|\frac{\mathscr{K} t_{1}^{\gamma}}{\gamma \Gamma(\gamma)}\right|<1
$$

and then prove the existence of a unique solution $f(x, t) \in$ $C^{0}\left[[a, b] \times\left[0, t_{1}\right]\right]$. To perform this, we can exploit the method of successive approximation by putting

$$
\begin{aligned}
& f_{0}(x, t)=f(x, 0), \\
& f_{n}(x, t)=f(x, 0)+\frac{1}{\Gamma(\gamma)} \int_{0}^{t} \frac{J\left(x, \xi, f_{n-1}(x, \xi)\right)}{(t-\xi)^{1-\gamma}} d \xi,
\end{aligned}
$$

$n \in \mathbb{N}$.

Obviously, $f(x, 0) \in C^{0}[0, T]$, and the differentiation of (30) with respect to $t$ leads to

$$
\partial_{t} f_{n}(x, t)=\frac{1}{\Gamma(\gamma-1)} \int_{0}^{t} \frac{J\left(x, \xi, f_{n-1}(x, \xi)\right)}{(t-\xi)^{-\gamma}} d \xi .
$$

Due to the fact that $f(x, 0)$ is differentiable with respect to $t$, then $f_{n}(x, t) \in C^{0}[0, T]$.

At this stage, we can estimate $\| f_{n}(x, t) \quad$ $f_{n-1}(x, t) \|_{C^{0}\left[[a, b] \times\left[0, t_{1}\right]\right]}$ for $n \in \mathbb{N}$; thus,

$$
\left\|f_{n}(x, t)-f_{n-1}(x, t)\right\|_{C^{0}\left[[a, b] \times\left[0, t_{1}\right]\right]} \leq \frac{\mathscr{K} t_{1}^{\gamma}}{\gamma \Gamma(\gamma)} .
$$

Also we have that

$$
\begin{aligned}
& \left\|f_{2}(x, t)-f_{1}(x, t)\right\|_{C^{0}\left[[a, b] \times\left[0, t_{1}\right]\right]} \\
& \quad \leq \| I_{0}^{\gamma} J\left(x, \xi, f_{1}(x, \xi)\right)
\end{aligned}
$$

$$
\begin{aligned}
& -J\left(x, \xi, f_{0}(x, \xi)\right)\left\|_{C^{0}\left[[a, b] \times\left[0, t_{1}\right]\right]} \leq \frac{t_{1}^{\gamma}}{\gamma \Gamma(\gamma)}\right\| f_{1}(x, t) \\
& -f_{0}(x, t) \|_{C^{0}\left[[a, b] \times\left[0, t_{1}\right]\right]} \leq \frac{\mathscr{K} t_{1}^{\gamma}}{\gamma \Gamma(\gamma)} \cdot \frac{t_{1}^{\gamma}}{\gamma \Gamma(\gamma)} .
\end{aligned}
$$

Proceeding by repeating the above steps $n$-times, we get the inequality

$$
\begin{gathered}
\left\|f_{2}(x, t)-f_{1}(x, t)\right\|_{C^{0}\left[[a, b] \times\left[0, t_{1}\right]\right]} \\
\leq\left(\frac{\mathscr{K} t_{1}^{\gamma}}{\gamma \Gamma(\gamma)}\right)^{n-1} \cdot \frac{t_{1}^{\gamma}}{\gamma \Gamma(\gamma)} .
\end{gathered}
$$

Hence, it happens that the sequence $\left\{f_{n}(x, t)\right\}_{n \in \mathbb{N}}$ approaches a limit function $f(x, t) \in C^{0}\left[[a, b] \times\left[0, t_{1}\right]\right]$. Also $\lim _{n \rightarrow \infty}\left\|f_{n}(x, t)-f_{n-1}(x, t)\right\|_{C^{0}\left[[a, b] \times\left[0, t_{1}\right]\right]}=0$ thanks to the assumption

$$
\left|\frac{t_{1}^{\gamma}}{\gamma \Gamma(\gamma)}\right|<1
$$

However, choosing $t_{1}=T$, we estimate

$$
J\left(x, t, f_{n}(x, \xi)\right)-J(x, t, f(x, t)) .
$$

Nevertheless, considering the Lipschitz condition of $J$, we have

$$
\begin{aligned}
& \| \frac{1}{\Gamma(\gamma)} \int_{0}^{t} \frac{J\left(x, \xi, f_{n}(x, \xi)\right)}{(t-\xi)^{1-\gamma}} d \xi-\frac{1}{\Gamma(\gamma)} \\
& \cdot \int_{0}^{t} \frac{J(x, \xi, f(x, \xi))}{(t-\xi)^{1-\gamma}} d \xi \|_{C^{0}[[a, b] \times[0, T]]} \leq\left(\frac{\mathscr{K} t_{1}^{\gamma}}{\gamma \Gamma(\gamma)}\right) \\
& \cdot\left\|f_{n}(x, t)-f(x, t)\right\|_{C^{0}\left[[a, b] \times\left[0, t_{1}\right]\right]}, \\
& \lim _{n \rightarrow \infty} \| \frac{1}{\Gamma(\gamma)} \int_{0}^{t} \frac{J\left(x, \xi, f_{n}(x, \xi)\right)}{(t-\xi)^{1-\gamma}} d \xi \quad=0 . \\
& -\frac{1}{\Gamma(\gamma)} \int_{0}^{t} \frac{J(x, \xi, f(x, \xi))}{(t-\xi)^{1-\gamma}} d \xi \|_{C^{0}[[a, b] \times[0, T]]}=0 .
\end{aligned}
$$

The equations obtained above prove that $f(x, t)$ is the solution of $(8)$ in the space $C^{0}[[a, b] \times[0, T]]$.

Uniqueness. Here we assume that it is possible to find two different solutions $f_{1}(x, t)$ and $f_{2}(x, t)$ verifying model $(8)$ on $\left[0, t_{1}\right]$ :

$$
\begin{aligned}
& \left\|f_{1}(x, t)-f_{2}(x, t)\right\|_{C^{0}\left[[a, b] \times\left[0, t_{1}\right]\right]} \\
& \quad \leq \frac{\mathscr{K} t_{1}^{\gamma}}{\gamma \Gamma(\gamma)}\left\|f_{1}(x, t)-f_{2}(x, t)\right\|_{C^{0}\left[[a, b] \times\left[0, t_{1}\right]\right]} .
\end{aligned}
$$

The above leads to $1 \leq \mathscr{K} t_{1}^{\gamma} / \gamma \Gamma(\gamma)$ and is a contradiction compared to the assumption. Thus, there is a unique solution $f(x, t) \in C^{0}\left[[a, b] \times\left[0, t_{1}\right]\right]$. 
Now, let us consider the closed interval $\left[t_{1}, t_{2}\right]$ so that $t_{2}=$ $t_{1}+h_{1}$ with $h_{1}>0$ and $t_{2}<T$. Hence, for $t \in\left[t_{1}, t_{2}\right]$, we get

$$
\begin{aligned}
f(x, t)= & \frac{1}{\Gamma(\gamma)} \int_{t_{1}}^{t} \frac{J\left(x, \xi, f_{n}(x, \xi)\right)}{(t-\xi)^{1-\gamma}} d \xi+f(x, 0) \\
& +\frac{1}{\Gamma(\gamma)} \int_{0}^{t_{1}} \frac{J\left(x, \xi, f_{n}(x, \xi)\right)}{(t-\xi)^{1-\gamma}} d \xi .
\end{aligned}
$$

Considering the above proof of uniqueness on $\left[0, t_{1}\right]$, we certainly have

$$
f(x, t)=f_{1}^{*}(x, t)+\frac{1}{\Gamma(\gamma)} \int_{t_{1}}^{t} \frac{J\left(x, \xi, f_{n}(x, \xi)\right)}{(t-\xi)^{1-\gamma}} d \xi
$$

where $f_{1}^{*}(x, t)=f(x, 0)+(1 / \Gamma(\gamma)) \int_{0}^{t_{1}}\left(J\left(x, \xi, f_{n}(x, \xi)\right) /(t-\right.$ $\left.\xi)^{1-\gamma}\right) d \xi$ is a known function.

The whole analysis and discussion presented above lead to the conclusion that there exists a unique solution $f(x, t) \in$ $C^{0}\left[[a, b] \times\left[t_{1}, t_{2}\right]\right]$ of $(8)$. Considering the interval $\left[t_{2}, t_{3}\right]$ so that $t_{3}=t_{2}+h_{2}$ with $h_{2}>0$ and $t_{3}<T$, it is possible to repeat the same steps as above and finally prove the existence of a unique solution $f(x, t) \in C^{0}[[a, b] \times[0, T]]$ of $(8)$ and, therefore, existence for $(6)$ in the space $C^{0, \gamma}[[a, b] \times[0, T]]$, which proves the theorem.

Theorem 3. Since J satisfies Theorem 1, then if

$$
\left|\frac{\mathscr{K} T^{\gamma}}{\gamma \Gamma(\gamma)}\right| \leq 1
$$

holds, then any sequence $f_{n}(x, t),(n \in \mathbb{N})$ as defined above is an approximation of the exact solution $f(x, t)$ with

$$
\begin{gathered}
\left\|f(x, t)-f_{n}(x, t)\right\|_{C^{0}\left[[a, b] \times\left[0, t_{1}\right]\right]} \\
\leq \frac{T^{\gamma}}{\gamma \Gamma(\gamma)} \frac{\mathscr{R}^{n}}{1-T^{\gamma} / \gamma \Gamma(\gamma)} .
\end{gathered}
$$

Proof. The proof is done by induction on $n$.

For

$$
\begin{aligned}
\left\|f_{1}(x, t)-f_{0}(x, t)\right\|_{C^{0}\left[[a, b] \times\left[0, t_{1}\right]\right]} & \leq \frac{\mathscr{K} T^{\gamma}}{\gamma \Gamma(\gamma)}, \\
\left\|f_{2}(x, t)-f_{1}(x, t)\right\|_{C^{0}\left[[a, b] \times\left[0, t_{1}\right]\right]} & \leq \mathscr{K}\left(\frac{T}{\gamma \Gamma(\gamma)}\right)^{2},
\end{aligned}
$$

recursively, we obtain

$$
\begin{gathered}
\left\|f_{n}(x, t)-f_{n-1}(x, t)\right\|_{C^{0}\left[[a, b] \times\left[0, t_{1}\right]\right]} \\
\leq \frac{T^{\gamma}}{\gamma \Gamma(\gamma)}\left(\frac{\mathscr{K} T^{\gamma}}{\gamma \Gamma(\gamma)}\right)^{n-1},
\end{gathered}
$$

but

$$
\begin{aligned}
\| f & (x, t)-f_{n}(x, t) \|_{C^{0}\left[[a, b] \times\left[0, t_{1}\right]\right]} \\
& =\lim _{j \rightarrow \infty}\left\|f_{n+j}(x, t)-f_{n}(x, t)\right\|_{C^{0}\left[[a, b] \times\left[0, t_{1}\right]\right]}
\end{aligned}
$$

$$
\begin{aligned}
= & \left\|f_{n+1}(x, t)-f_{n}(x, t)\right\|_{C^{0}\left[[a, b] \times\left[0, t_{1}\right]\right]} \\
& +\left\|f_{n+2}(x, t)-f_{n+1}(x, t)\right\|_{C^{0}\left[[a, b] \times\left[0, t_{1}\right]\right]}+\cdots \\
\leq & \mathscr{K}^{n}\left(\frac{T^{\gamma}}{\gamma \Gamma(\gamma)}\right)^{n+1}+\mathscr{K}^{n+1}\left(\frac{T^{\gamma}}{\gamma \Gamma(\gamma)}\right)^{n+2}+\cdots \\
= & \mathscr{K}^{n}\left(\frac{T^{\gamma}}{\gamma \Gamma(\gamma)}\right)^{n+1}\left[\sum_{k=0}^{\infty}\left(\frac{\mathscr{K} T^{\gamma}}{\gamma \Gamma(\gamma)}\right)^{k}\right]
\end{aligned}
$$

and the proof is complete.

The above theorem also shows a rapid convergence of $f_{n}(x, t)$ to $f(x, t)$.

\section{Analysis Using the Caputo-Fabrizio Fractional Derivative (CFD)}

3.1. Definition and Preliminary Concepts. Let $(E,\|\|)$ be a Banach space and consider $\mathscr{K}$ as a self-map of $E$. Let $\delta_{n+1}=$ $g\left(\mathscr{K}, \delta_{n}\right)$ be a recursive method where we assume that $F(\mathscr{K})$ is a set representing all the fixed points of the map $\mathscr{K}$ and is assumed to have at least one element. Assume that $\delta_{n}$ tends to an element $s \in F(\mathscr{K})$. Let $\left\{e_{n}\right\} \subseteq E$ and define $d_{n}=$ $\left\|e_{n+1}-g\left(\mathscr{K}, e_{n}\right)\right\|$.

$\lim _{n \rightarrow \infty} d_{n}=0$ implies that $\lim _{n \rightarrow \infty} e_{n}=s$. Then, with the above settings, we say that the recursive relation $\delta_{n+1}=$ $g\left(\mathscr{K}, \delta_{n}\right)$ is $\mathscr{K}$-stable. Moreover, with the assumption that $\left\{e_{n}\right\}$ has an upper boundary and if all the conditions above hold for $\delta_{n+1}=\mathscr{K} \delta_{n}$, then it is called Picard's iteration and is $\mathscr{K}$ stable.

Lemma 4 (see [24]). Let (E, \|\|) be a Banach space and $\mathscr{K}$ a self-map of E satisfying

$$
\|\mathscr{K} x-\mathscr{K} y\| \leq C\|x-\mathscr{K} x\|+C\|x-y\|
$$

for all $x, y \in E$, where $0 \leq C, 0 \leq \gamma \leq 1$. With the assumption that $\mathscr{K}$ has a fixed point q, then $\mathscr{K}$ is Piccard $\mathscr{K}$-stable.

\subsection{Analysis}

Theorem 5. Consider the self-map $\mathscr{K}$ and redefine model (6) as the recurrence relation:

$$
\begin{aligned}
& \mathscr{K}\left(f_{n}(x, t)\right)=f_{n+1}(x, t)=f_{n}(x, t) \\
& \quad+{ }^{c f} I_{t}^{\gamma}\left[-f_{x x x x x}(x, \xi)-15 f(x, \xi) f_{x x x}(x, \xi)\right. \\
& \left.\quad-15 f_{x x}(x, \xi) f_{x}(x, \xi)-45 f^{2}(x, \xi) f_{x}(x, \xi)\right] .
\end{aligned}
$$

Then, it is $\mathscr{K}$-stable in $L^{2}(a, b)$. 
Proof. We start by showing that $\mathscr{K}$ has a fixed-point. Let $i, j \in$ $\mathbb{N}$; then,

$$
\begin{aligned}
\| \mathscr{K} & f_{i}(x, t)-\mathscr{K} f_{j}(x, t)\|=\| f_{i+1}(x, t)-f_{j+1}(x, t) \| \\
& =\| f_{i}(x, t)+{ }^{c f} I_{t}^{\gamma}\left[-\partial_{x^{5}}^{5} f_{i}-15 f_{i} \partial_{x^{3}}^{3} f_{i}\right. \\
& \left.-15 \partial_{x^{2}}^{2} f_{i} \partial_{x} f_{i}-45 f_{i}^{2} \partial_{x} f_{i}\right]-f_{j}(x, t) \\
& +{ }^{c f} I_{t}^{\gamma}\left[\partial_{x^{5}}^{5} f_{j}-15 f_{j} \partial_{x^{3}}^{3} f_{j}-15 \partial_{x^{2}}^{2} f_{j} \partial_{x} f_{j}\right. \\
& \left.-45 f_{j}^{2} \partial_{x} f_{j}\right] \|,
\end{aligned}
$$

where

$$
\begin{aligned}
{ }^{c f} I_{t}^{\gamma} f(t)= & \frac{2(1-\gamma)}{(2-\gamma) M(\gamma)} f(t) \\
& +\frac{2 \gamma}{(2-\gamma) M(\gamma)} \int_{0}^{t} f(\xi) d \xi
\end{aligned}
$$

is the fractional integral (antiderivative) associated with the Caputo-Fabrizio fractional derivative defined in (4). Then,

$$
\begin{aligned}
& \left\|\mathscr{K} f_{i}(x, t)-\mathscr{K} f_{j}(x, t)\right\| \\
& \leq\left\|f_{i}(x, t)-f_{j}(x, t)\right\|+\left\|c f I_{t}^{\gamma}\left[\partial_{x^{5}}^{5}\left(f_{i}-f_{j}\right)\right]\right\| \\
& -15\left\|c f I_{t}^{\gamma}\left[f_{i} \partial_{x^{3}}^{3} f_{i}-f_{j} \partial_{x^{3}}^{3} f_{j}\right]\right\| \\
& -15\left\|{ }^{c f} I_{t}^{\gamma}\left[\left(\partial_{x^{2}}^{2} f_{i} \partial_{x} f_{i}-\partial_{x^{2}}^{2} f_{j} \partial_{x} f_{j}\right)\right]\right\|, \\
& 30\left\|^{c f} I_{t}^{\gamma}\left[\left(f_{i}^{2} \partial_{x} f_{i}-f_{j}^{2} \partial_{x} f_{j}\right)\right]\right\| \\
& \leq \frac{2(1-\gamma)}{(2-\gamma) M(\gamma)} \kappa_{1} \kappa_{2} \kappa_{3} \kappa_{4} \kappa_{5}\left\|f_{i}-f_{j}\right\| \\
& +\frac{2 \gamma}{(2-\gamma) M(\gamma)} \kappa_{1} \kappa_{2} \kappa_{3} \kappa_{4} \kappa_{5} T\left\|f_{i}-f_{j}\right\| \\
& +\frac{30(1-\gamma)}{(2-\gamma) M(\gamma)} \delta_{1} \delta_{2} \delta_{3}\left\|f_{i}-f_{j}\right\| \\
& +\frac{-45 \gamma}{(2-\gamma) M(\gamma)} \delta_{1} \delta_{2} \delta_{3} T\left\|f_{i}-f_{j}\right\| \\
& +\frac{15(1-\gamma)}{(2-\gamma) M(\gamma)} \vartheta_{1} \mathscr{K}_{1}\left\|f_{i}-f_{j}\right\| \\
& +\frac{15}{(2-\gamma) M(\gamma)} \vartheta_{1} \mathscr{K}_{1} T\left\|f_{i}-f_{j}\right\| \\
& +\frac{90(1-\gamma)}{3(2-\gamma) M(\gamma)} \delta_{1} A_{1}\left\|f_{i}-f_{j}\right\| \\
& +\frac{90}{3(2-\gamma) M(\gamma)} \delta_{1} A_{1} T\left\|f_{i}-f_{j}\right\|,
\end{aligned}
$$

where the Lipschitz condition for the differential operator $\partial_{x}$ has been used together with the following positive constants $\kappa_{i}, i=1,2,3,4,5 ; \delta_{i}, i=1,2,3$; and $\vartheta_{1}$ and $\delta_{1}$. Then,

$$
\left\|\mathscr{K} f_{i}(x, t)-\mathscr{K} f_{j}(x, t)\right\| \leq R\left\|f_{i}(x, t)-f_{j}(x, t)\right\|,
$$

where

$$
\begin{aligned}
R= & \frac{2(1-\gamma)}{(2-\gamma) M(\gamma)} \kappa_{1} \kappa_{2} \kappa_{3} \kappa_{4} \kappa_{5} \\
& +\frac{2 \gamma}{(2-\gamma) M(\gamma)} \kappa_{1} \kappa_{2} \kappa_{3} \kappa_{4} \kappa_{5} T \\
& +\frac{-45(1-\gamma)}{(2-\gamma) M(\gamma)} \delta_{1} \delta_{2} \delta_{3}+\frac{-45 \gamma}{(2-\gamma) M(\gamma)} \delta_{1} \delta_{2} \delta_{3} T \\
& +\frac{25(1-\gamma)}{(2-\gamma) M(\gamma)} \vartheta_{1} \mathscr{K}_{1}+\frac{25}{(2-\gamma) M(\gamma)} \vartheta_{1} \mathscr{K}_{1} T \\
& +\frac{40(1-\gamma)}{3(2-\gamma) M(\gamma)} \delta_{1} A_{1} \\
& +\frac{40}{3(2-\gamma) M(\gamma)} \delta_{1} A_{1} T
\end{aligned}
$$

and this proves that the Lipschitz condition holds for the nonlinear operator $\mathscr{K}$ and hence, it has a fixed point.

Lastly, if we take $C=0$ and $C=R$, then the conditions of Lemma 4 hold for $\mathscr{K}$ which is therefore Piccard $\mathscr{K}$-stable and the proof is complete.

Remark 6. The previous theorem shows the existence of a unique solution for model (6) obtained via the fixed point of $\mathscr{K}$ in the recurrence relation (47).

\section{Numerical Schemes}

Numerical methods are performed in this section for model with both derivatives. Before that, we show how the solutions can be derived for both types of models with the use of some recursive techniques together with Laplace transform (for the model with CD) and Sumudu transform (for the model with CFD).

4.1. Model with Caputo Derivative. To proceed with the solvability, consider model (6) and apply the Laplace transform $\mathscr{L}$ on both sides. We obtain

$$
\begin{aligned}
p f & (x, p)-f(x, 0)=\mathscr{L}\left[-f_{x x x x x}(x, t)\right. \\
& -15 f(x, t) f_{x x x}(x, t)-15 f_{x}(x, t) f_{x x}(x, t) \\
& \left.-45 f^{2}(x, t) f_{x}(x, t)\right](p) ;
\end{aligned}
$$


equivalently,

$$
\begin{aligned}
& f(x, p)=\frac{f(x, 0)}{p}+\frac{1}{p} \mathscr{L}\left[-f_{x x x x x}(x, t)\right. \\
& -15 f(x, t) f_{x x x}(x, t)-15 f_{x}(x, t) f_{x x}(x, t) \\
& \left.\quad-45 f^{2}(x, t) f_{x}(x, t)\right](p) .
\end{aligned}
$$

The inverse Laplace transform $\mathscr{L}^{-1}$ gives

$$
\begin{aligned}
& f(x, t)=f(x, 0)+\mathscr{L}^{-1}\left(\frac { 1 } { p } \mathscr { L } \left[-f_{x x x x x}(x, t)\right.\right. \\
& -15 f(x, t) f_{x x x}(x, t)-15 f_{x}(x, t) f_{x x}(x, t) \\
& \left.\left.-45 f^{2}(x, t) f_{x}(x, t)\right]\right)(t) .
\end{aligned}
$$

At this stage we can introduce the following iterative system:

$$
\begin{aligned}
& f_{0}(x, t)=f(x, 0), \\
& f_{n+1}(x, t)=f_{n}(x, t)+\mathscr{L}^{-1}\left(\frac { 1 } { p } \mathscr { L } \left[-f_{x x x x x}(x, t)\right.\right. \\
& \quad-15 f(x, t) f_{x x x}(x, t)-15 f_{x}(x, t) f_{x x}(x, t) \\
& \left.\left.\quad-45 f^{2}(x, t) f_{x}(x, t)\right]\right)(t) .
\end{aligned}
$$

This system is exploited to determine an approximation for the solution of the model with Caputo derivative. The stability of the method is well known since it uses the fixed-point theorem. Whence, this approximation reads as

$$
f(x, t)=\lim _{n \rightarrow \infty} f_{n}(x, t) .
$$

4.2. Model with Caputo-Fabrizio Derivative. Similarly, to proceed with the solvability, we will consider model (6) and apply the Sumudu transform on both sides. But first of all, we have to recall the well-known relation that exists between the Sumudu transform $\mathscr{W}$ and the Caputo-Fabrizio derivative given as

$$
\mathscr{W}\left({ }_{0}^{c f} D_{t}^{\gamma} f(t)\right)=M(\gamma) \frac{p S(p)-f(o)}{1-\gamma-\gamma p},
$$

where $S(p)=\mathscr{W}(f(t))$ is the Sumudu transform of $f(t)$. Applying the Sumudu transform $\mathscr{W}$ on both sides of (6) gives

$$
\begin{aligned}
& \frac{p f(x, p)-f(x, 0)}{1-\gamma-\gamma p}=\mathscr{W}\left[-f_{x x x x x}(x, t)\right. \\
& -15 f(x, t) f_{x x x}(x, t)-15 f_{x}(x, t) f_{x x}(x, t) \\
& \left.\quad-45 f^{2}(x, t) f_{x}(x, t)\right](p) .
\end{aligned}
$$

After rearrangement

$$
\begin{aligned}
& f(x, p)=\frac{f(x, 0)}{p}+(1-\gamma-\gamma p) \mathscr{W}\left[-f_{x x x x x}(x, t)\right. \\
& -15 f(x, t) f_{x x x}(x, t)-15 f_{x}(x, t) f_{x x}(x, t) \\
& \left.-45 f^{2}(x, t) f_{x}(x, t)\right](p) .
\end{aligned}
$$

The inverse Sumudu transform $\mathscr{W}^{-1}$ leads to

$$
\begin{aligned}
& f(x, t)=f(x, 0)+\mathscr{W}^{-1}((1-\gamma-\gamma p) \\
& \cdot \mathscr{W}\left[-f_{x x x x x}(x, t)-15 f(x, t) f_{x x x}(x, t)\right. \\
& \left.\left.\quad-15 f_{x}(x, t) f_{x x}(x, t)-45 f^{2}(x, t) f_{x}(x, t)\right]\right)(t) .
\end{aligned}
$$

Similarly as above, we can introduce the following iterative system:

$$
\begin{aligned}
& f_{0}(x, t)=f(x, 0), \\
& f_{n+1}(x, t)=f_{n}(x, t)+\mathscr{W}^{-1}((1-\gamma-\gamma p) \\
& \quad \cdot \mathscr{W}\left[-f_{x x x x x}(x, t)-15 f(x, t) f_{x x x}(x, t)\right. \\
& \left.\left.\quad-15 f_{x}(x, t) f_{x x}(x, t)-45 f^{2}(x, t) f_{x}(x, t)\right]\right)(t) .
\end{aligned}
$$

Again, this system is exploited to determine an approximation for the solution of the Caputo-Fabrizio model. The stability of the method is well known and is established using the fixed-point theorem and the approximation is given by

$$
f(x, t)=\lim _{n \rightarrow \infty} f_{n}(x, t) .
$$

Exploiting the recurrence schemes above, numerical simulations for models with both derivatives, namely, the Caputo derivative (CD) and Caputo-Fabrizio derivative (CFD), are depicted in Figure 1 for different values of the derivative order $\gamma$ equal to 1 and 0.80 . There are clear similarities in the observed behaviors in Figure 1(a) (model with the CD) and Figure 1(b) (model with the CFD) both represented for $\gamma=$ 1.00. We note existence of the standard multisoliton solutions well known to be related to Sawada-Kotera equation. The same analysis holds for Figure 1(c) (model with the CD) and Figure 1(d) (model with the CFD) all drawn for $\gamma=0.80$.

\section{Concluding Remarks}

The prime goal of this work has been to exploit the concept of Piccard $\mathscr{K}$-stability as well as various fixed-point theories, Sumudu and Laplace transforms to address the solvability of the nonlinear Sawada-Kotera model expressed with both Caputo and Caputo-Fabrizio derivatives with fractional order. The analysis performed in this paper has enabled us to show existence and uniqueness results for both models and compare the behaviors of their solutions. As expected, numerical simulations done for some values of the derivative order $\gamma$ show similar behaviors of the solutions and point out existence of multisoliton solutions for both equations. This 


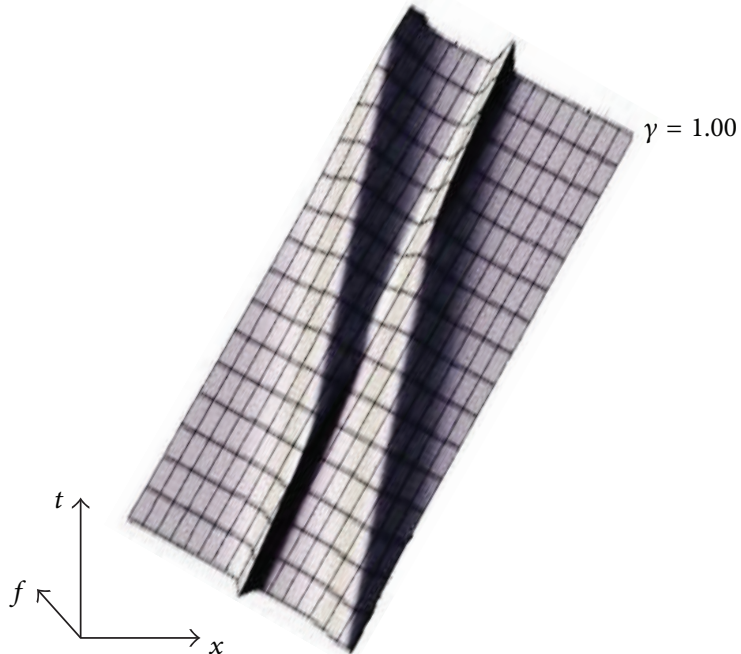

(a) Caputo representation

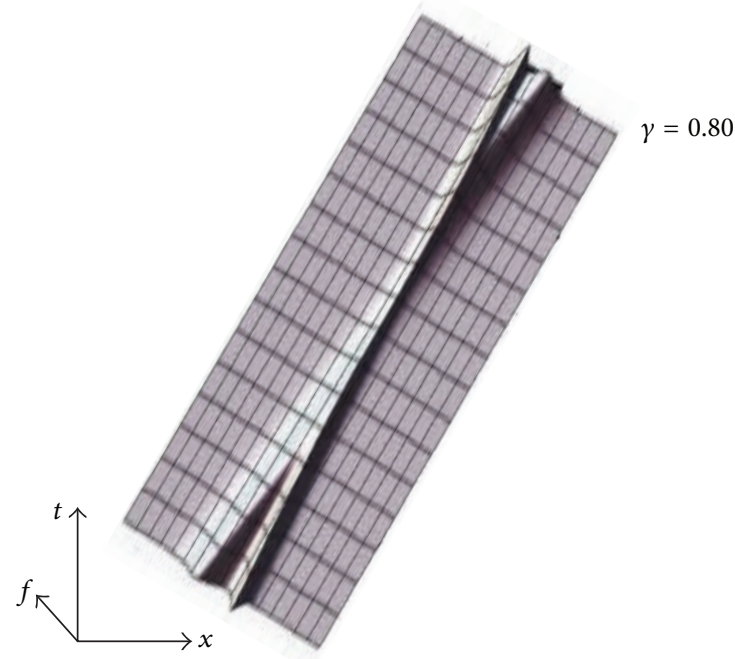

(c) Caputo representation

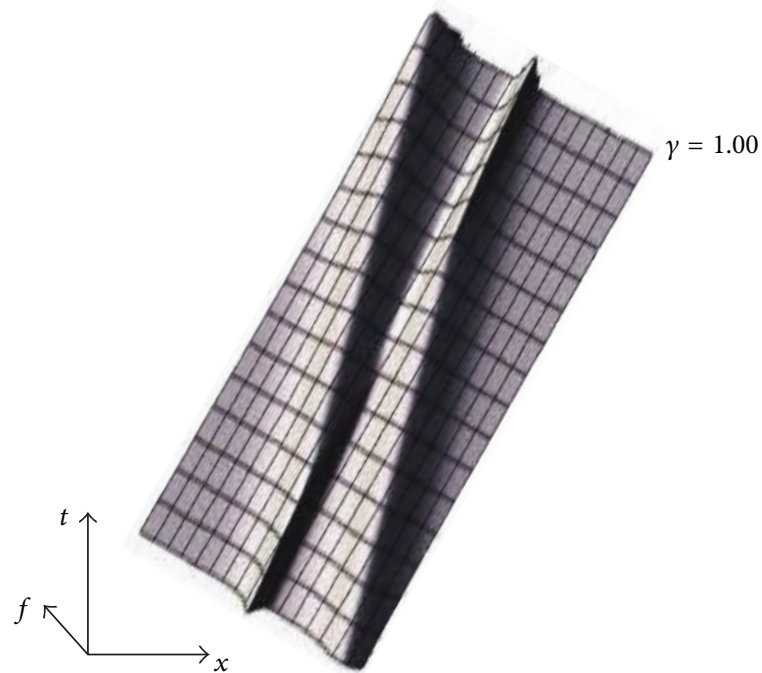

(b) Caputo-Fabrizio representation

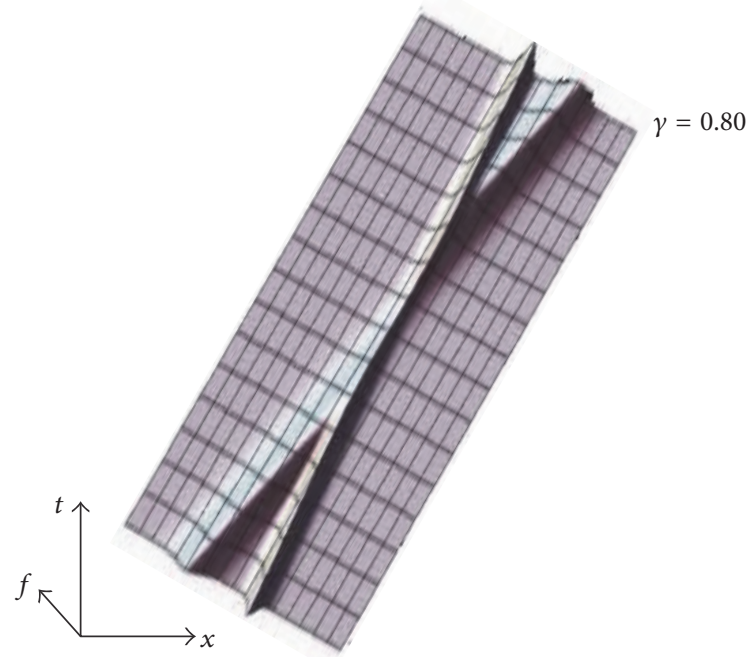

(d) Caputo-Fabrizio representation

FIGURE 1: Representation of the solution $f(x, t)$ when $\gamma=1.00$ in (a) and (b) and $\gamma=0.80$ in (c) and (d) with both Caputo derivative and Caputo-Fabrizio derivative. As expected, similar behaviors are pointed out in each case and we can see the existence of multisoliton solutions for both types of equations.

comparative analysis is more than useful in the sense that it validates the use of any of both derivatives in the description of natural phenomena like those related to shallow water waves or ion-acoustic waves and will surely yield more similar and complex investigations within the scientific community.

\section{Competing Interests}

The authors declare that there is no conflict of interests regarding the publication of this paper.

\section{References}

[1] E. F. Doungmo Goufo, "Stability and convergence analysis of a variable order replicator-mutator process in a moving medium," Journal of Theoretical Biology, vol. 403, pp. 178-187, 2016.

[2] J. T. Machado, F. Mainardi, and V. Kiryakova, "Fractional calculus: quo vadimus? (Where are we going?)," Fractional Calculus and Applied Analysis, vol. 18, no. 2, pp. 495-526, 2015.

[3] S. Kumar and M. M. Rashidi, "New analytical method for gas dynamics equation arising in shock fronts," Computer Physics Communications, vol. 185, no. 7, pp. 1947-1954, 2014.

[4] Y. Khan, K. Sayevand, M. Fardi, and M. Ghasemi, "A novel computing multi-parametric homotopy approach for system of linear and nonlinear Fredholm integral equations," Applied Mathematics and Computation, vol. 249, pp. 229-236, 2014.

[5] M. Caputo, "Linear models of dissipation whose Q is almost frequency independent-II," Geophysical Journal International, vol. 13 , no. 5, pp. 529-539, 1967. 
[6] A. A. Kilbas, H. M. Srivastava, and J. J. Trujillo, Theory and Applications of Fractional Differential Equations, vol. 204, Elsevier Science, Amsterdam, The Netherlands, 2006.

[7] E. F. D. Goufo, "Application of the Caputo-Fabrizio fractional derivative without singular kernel to Korteweg-de VriesBurgers equation," Mathematical Modelling and Analysis, vol. 21, no. 2, pp. 188-198, 2016.

[8] E. F. Doungmo Goufo, "A biomathematical view on the fractional dynamics of cellulose degradation," Fractional Calculus and Applied Analysis, vol. 18, no. 3, pp. 554-564, 2015.

[9] Y. Luchko and R. Gorenflo, The Initial Value Problem for Some Fractional Differential Equations with the Caputo Derivative, Series A0898, Fachbreich Mathematik and Informatik, Freic Universitat Berlin, 1998.

[10] S. Kumar, A. Kumar, and I. K. Argyros, "A new analysis for the Keller-Segel model of fractional order," Numerical Algorithms, 2016.

[11] I. Area, H. Batarfi, J. Losada, J. J. Nieto, W. Shammakh, and Á. Torres, "On a fractional order Ebola epidemic model," Advances in Difference Equations, vol. 2015, no. 1, p. 278, 2015.

[12] A. Rachah and D. F. M. Torres, "Mathematical modelling, simulation, and optimal control of the 2014 Ebola outbreak in West Africa," Discrete Dynamics in Nature and Society, vol. 2015, Article ID 842792, 9 pages, 2015.

[13] M. Du, Z. Wang, and H. Hu, "Measuring memory with the order of fractional derivative," Scientific Reports, vol. 3, Article ID 3431, 2013.

[14] S. Kumar, D. Kumar, and J. Singh, "Fractional modelling arising in unidirectional propagation of long waves in dispersive media," Advances in Nonlinear Analysis, vol. 5, no. 4, pp. 383394, 2016.

[15] M. Caputo and M. Fabrizio, "A new definition of fractional derivative without singular kernel," Progress in Fractional Differentiation and Applications, vol. 1, no. 2, pp. 1-13, 2015.

[16] J. Losada and J. J. Nieto, "Properties of the new fractional derivative without singular Kernel," Progress in Fractional Differentiation and Applications, vol. 1, no. 2, pp. 87-92, 2015.

[17] A. Atangana and D. Baleanu, "New fractional derivatives with nonlocal and non-singular kernel: theory and application to heat transfer model," Thermal Science, vol. 20, no. 2, pp. 763769, 2016.

[18] E. F. Doungmo Goufo, "Chaotic processes using the twoparameter derivative with non-singular and non-local kernel: basic theory and applications," Chaos: An Interdisciplinary Journal of Nonlinear Science, vol. 26, no. 8, 2016.

[19] R. Beals and R. R. Coifman, "Scattering and inverse scattering for first order systems," Communications on Pure and Applied Mathematics, vol. 37, no. 1, pp. 39-90, 1984.

[20] V. S. Gerdjikov and A. B. Yanovski, "Completeness of the eigenfunctions for the caudrey-beals-coifman system," Journal of Mathematical Physics, vol. 35, no. 7, pp. 3687-3725, 1994.

[21] Z. Yan, "The Riccati equation with variable coefficient expansion algorithm to find more exact solutions of nonlinear differential equations," Journal of Nonlinear Mathematical Physics, vol. 7, pp. 1-3, 2003.

[22] P. R. Massopust and A. I. Zayed, "On the invalidity of Fourier series expansions of fractional order," Fractional Calculus and Applied Analysis, vol. 18, no. 6, pp. 1507-1517, 2015.

[23] M. Caputo, J. M. Carcione, and M. A. B. Botelho, "Modeling extreme-event precursors with the fractional diffusion equation," Fractional Calculus and Applied Analysis, vol. 18, no. 1, pp. 208-222, 2015.
[24] D. Eberly, Stability Analysis for Systems of Differential Equations, Geometric Tools LLC, 2008, http://www.geometrictools.com. 


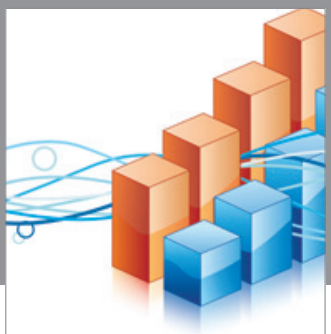

Advances in

Operations Research

vatem alat4

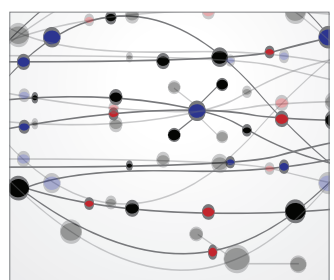

\section{The Scientific} World Journal
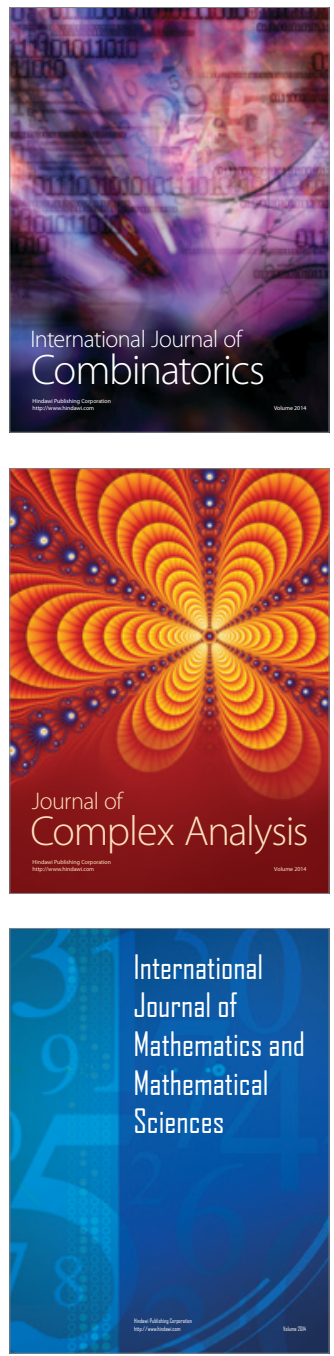
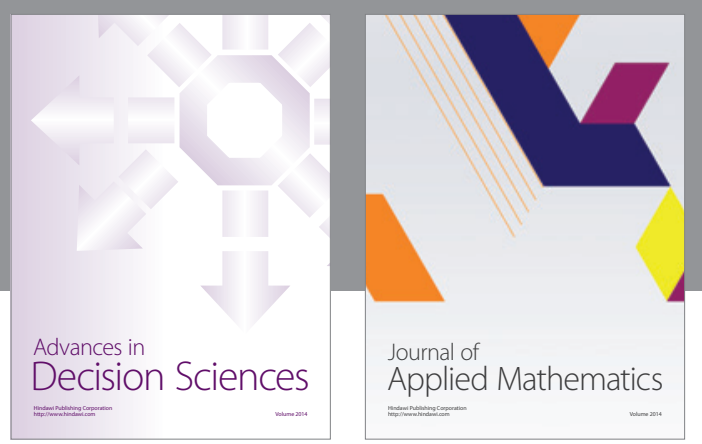

Algebra

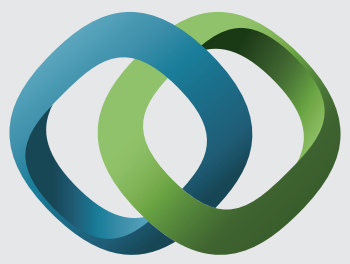

\section{Hindawi}

Submit your manuscripts at

https://www.hindawi.com
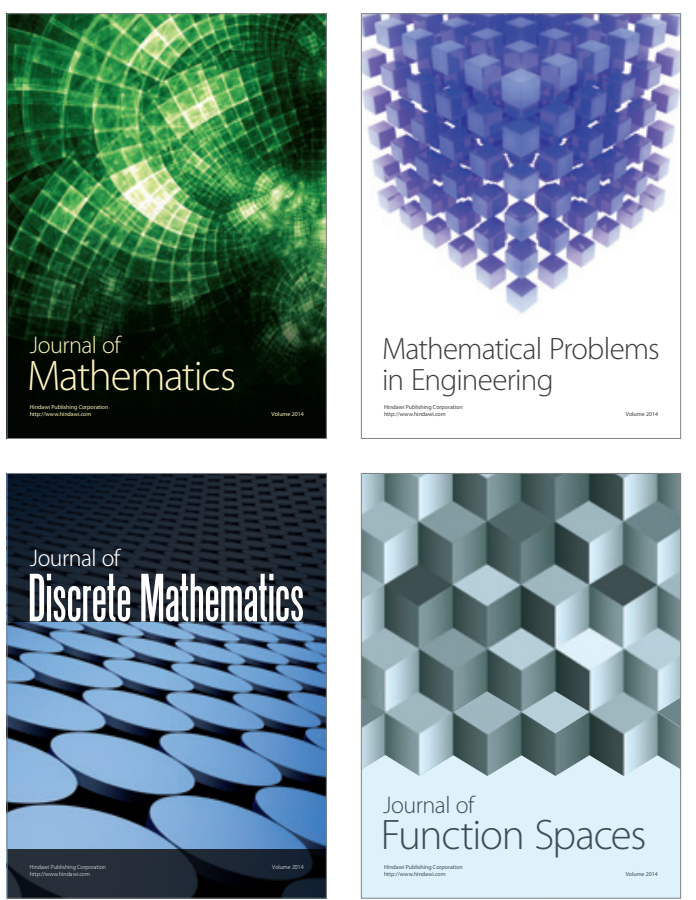

Mathematical Problems in Engineering
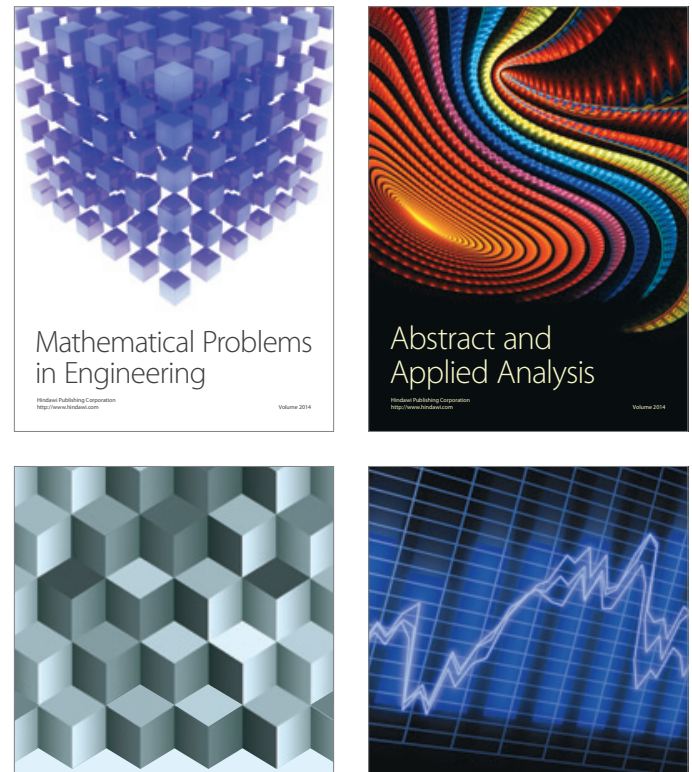

Journal of

Function Spaces

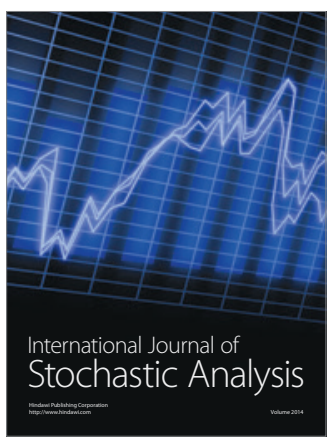

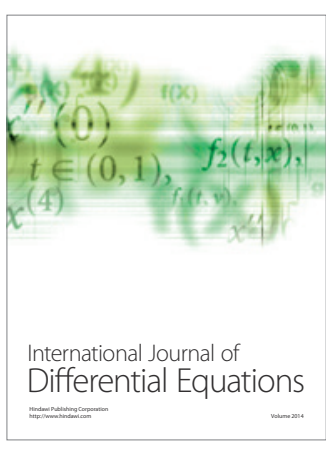
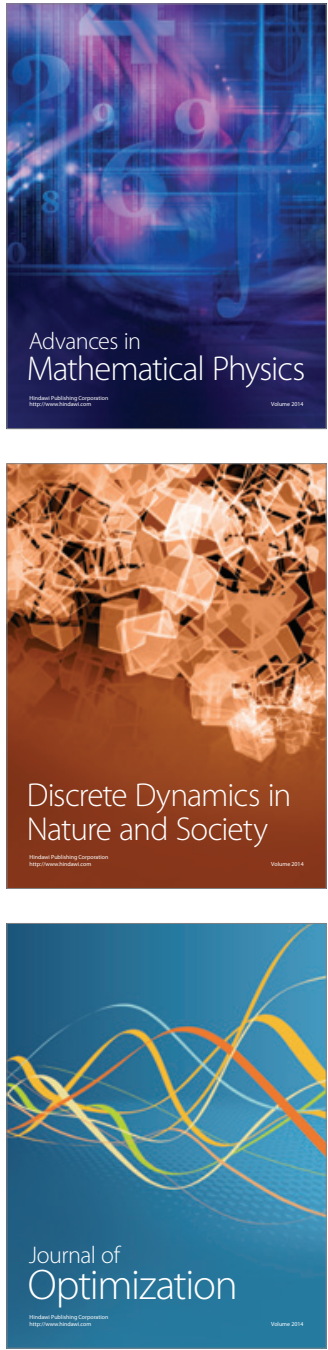